\title{
EFPI is an ER stress-induced glycoprotein which interacts with the pro-apoptotic protein Par-4
}

This article was published in the following Dove Press journal:

Cell Health and Cytoskeleton

8 May 2009

Number of times this article has been viewed

\author{
Sarah Appel 1,2,6 \\ Susanne Vetterkind ${ }^{1,2,6}$ \\ Ansgar Koplin ${ }^{1,3}$ \\ Barbara Maertens 1,4 \\ Meike Boosen ${ }^{1,5}$ \\ Ute Preuss' \\ 'The Institute of Genetics, University \\ of Bonn, Bonn, Germany; 'Department \\ of Health Sciences, Sargent College of \\ Health and Rehabilitation Sciences, \\ Boston University, Boston, MA, \\ USA; ${ }^{3}$ Center for Molecular Biology \\ Heidelberg (ZMBH), Heidelberg, \\ Germany; ${ }^{4}$ Institute of Biochemistry II, \\ University of Cologne, Cologne, \\ Germany; ${ }^{5}$ Institute of Pharmacology \\ and Toxicology, University Hospital \\ of Johann Wolfgang Goethe-University \\ Frankfurt am Main, Frankfurt am Main, \\ Germany; ${ }^{6}$ These authors contributed \\ equally to this work
}

Correspondence: Ute Preuss Institute of Genetics, University of Bonn, Roemerstr. 164, D-53117 Bonn, Germany Tel +49228736842

Fax +49228734263

Email u.preuss@uni-bonn.de

\begin{abstract}
We have isolated the rat ortholog of EFP1 (EF-hand binding protein 1) as a novel interaction partner of the pro-apoptotic protein Par-4 (prostate apoptosis response-4). Rat EFP1 contains two thioredoxin domains, the $\mathrm{COOH}$-terminal one harboring a CGFC motif, and has a similar protein domain structure as members of the protein disulfide isomerase (PDI) family. In REF52.2 and CHO cells, EFP1 colocalized with the endoplasmic reticulum (ER) marker PDI. Furthermore, EFP1 possesses catalytic activity as demonstrated by an insulin disulfide reduction assay. Western blot analysis revealed two EFP1 protein bands of approximately 136 and $155 \mathrm{kDa}$, representing different glycosylation states of the protein. Complex formation between EFP1 and Par-4 was confirmed in vitro and in vivo by co-immunoprecipitation, dot blot overlay and pull-down experiments. In CHO cells, coexpression of EFP1 and Par-4 resulted in enhanced Par-4-mediated apoptosis, which required the catalytic activity of EFP1. Interestingly, EFP1 was specifically upregulated in NIH3T3 cells after induction of ER stress by thapsigargin, tunicamycin, and brefeldin A, but not by agents that induce oxidative stress or ER-independent apoptosis. Furthermore, we could show that the induction of apoptosis by $\mathrm{Ca}^{2+}$ stress-inducing agents was significantly decreased after siRNA oligonucleotide-mediated knockdown of Par-4. Our data suggest that EFP1 might represent a cell-protective enzyme that could play an important role in the decision between survival and initiation of Par-4-mediated apoptosis.
\end{abstract}

Keywords: Par-4, EFP1, PDI, thioredoxin, ER stress, apoptosis

\section{Introduction}

Conditions that alter the endoplasmic reticulum (ER) environment, commonly referred to as ER stress, can affect the efficiency of ER enzymes in performing their multiple tasks like protein folding and glycosylation. For example, extraordinarily high protein synthesis rates, as they occur in a virus-infected cell, can lead to an ER overload and to an accumulation of misfolded or unfolded proteins in the ER. Likewise, deregulated calcium homeostasis can disturb the function of the ER. In order to overcome such situations, genetic and molecular programs are activated to restore the function of the ER. A typical ER stress response initially consists of the attenuation of translation and activation of protein degradation pathways, while simultaneously the specific ER stress transcription factor ATF6 is activated, ${ }^{1-3}$ which upregulates genes for ER chaperones like protein disulfide isomerase (PDI) and glucose-regulated protein 78/immunoglobulin binding protein (GRP78/BiP) to cope with the enhanced protein charge. ${ }^{4,5}$ Many stress-inducible ER chaperones, including PDI, glucose-regulated protein 94 (GRP94) and GRP78/BiP, also contain calcium

submit your manuscript | www.dovepress.coin 
binding domains that help maintaining or restoring the calcium gradient between the ER and the cytoplasm. ${ }^{6-8}$

However, when the ER stress response fails to overcome the situation, signaling pathways are activated that induce execution of the programmed cell death, apoptosis. ${ }^{9}$ To date, two major ER-specific apoptotic pathways are known. One of them initiates apoptosis by activation of caspases, cysteine-aspartate proteinases that play a central role in apoptosis. ${ }^{10}$ Caspase-12 and caspase- 4 have been described as ER-specific caspases that are activated during persistent ER stress. ${ }^{11,12}$ In addition, participation of other caspases has been suggested. ${ }^{13,14}$ A recent publication has demonstrated the dispensability of caspase- 4 and caspase- 12 for caspasedependent ER stress-mediated apoptosis, ${ }^{15}$ suggesting the participation of other caspases. Thus, multiple redundant pathways may lead from ER stress to caspase-activation. ER stress-induced apoptosis can also be initiated independently of caspases, since an alternative apoptotic pathway in ER stress is triggered by GADD153/CHOP, an ER stressinducible transcription factor. ${ }^{16}$ GADD153/CHOP alters the balance between the anti-apoptotic protein $\mathrm{Bcl}-2$ and the pro-apoptotic Bax, ${ }^{17}$ resulting in cytochrome $\mathrm{C}$ release from the mitochondria, a highly conserved pathway of apoptosis induction. ${ }^{18}$

One of the proteins that has been implicated in ER stress-dependent apoptotic processes is the pro-apoptotic protein Par-4 (prostate apoptosis response-4). ${ }^{19}$ The protein was originally discovered as the product of an early response gene in ionomycin-induced apoptosis of prostate cancer cells. ${ }^{20}$ The mechanisms how Par-4 can induce or support apoptotic processes are versatile. We have demonstrated that Par-4 can bind to actin filaments and can induce apoptosis by sequestering nuclear proteins to the cytoskeleton like the pro-apoptotic serine/threonine kinase Dlk/ZIPK or the pro-apoptotic protein Amida. ${ }^{21-23}$ In other cellular settings, Par-4 can act as a transcriptional co-repressor of the $b c l-2$ gene, ${ }^{24}$ or as an inhibitor of PKB/Akt. ${ }^{25}$ However, many aspects of Par-4-mediated apoptosis are still poorly understood. In an effort to identify novel interaction partners of Par-4 that might serve as regulators or downstream effectors in Par-4-mediated apoptosis, we identified the rat ortholog of EF-hand binding protein 1 (EFP1) in a yeast two-hybrid screen. EFP1 was previously identified as an interaction partner of Duox, a protein involved in $\mathrm{H}_{2} \mathrm{O}_{2}$ production in the thyroid. ${ }^{26}$ We show that EFP1 is an ER-resident glycoprotein which possesses enzymatic activity in an insulin reduction assay and demonstrate that EFP1 is upregulated during ER stress. Coexpression of EFP1 and Par-4 resulted in enhanced
EFP1/Par-4-mediated apoptosis, suggesting that EFP1 can trigger Par-4-mediated apoptosis.

\section{Materials and methods Generation of the cDNA library and yeast two-hybrid screening}

The generation of the cDNA library and the yeast two-hybrid screening have been described previously. ${ }^{27}$ Briefly, poly $(\mathrm{A})^{+}$RNA was isolated from TZ103 cells, reversetranscribed, and inserted into the HybriZAPTM vector ${ }^{28}$ using the ZAPExpress ${ }^{\mathrm{TM}}$ cDNA synthesis kit (Stratagene, La Jolla, CA). Two-hybrid screening was performed with Par-4 fused to the GAL4 DNA binding domain as bait. His and lacZ-positive clones were isolated and the cDNA inserts were further analyzed by cloning and sequencing.

\section{Cloning of rat EFPI cDNA and construction of plasmids}

The rat EFP1 cDNA was amplified by RT-PCR using the E14-2 cDNA as template and the oligonucleotides 5'-GAAGGTCAAGTATCCAGGAGGCTGG-3' and 5'-CCCAAGCTTATGTCGGAATGCGGA-3' derived from the rat sequence EST XM220122.2. A C-terminally GFP-tagged full-length EFP1 cDNA construct (denoted EFP1-GFP) was generated by PCR with oligonucleotides 5' -CGGGA TCCTGATGGCGCGCCAG-3' and 5'-GCGGATCCAGTAAGTCTGTCCTGTTCTC-3' and subcloned into the pEGFP-N1 expression vector using BamHI restriction sites (Clontech, Heidelberg, Germany). In order to generate a thioredoxin-domain II mutant of EFP1 (denoted EFP1 ${ }^{\text {THX-}}$ ) in which the cysteine residues of the CXXC motif were replaced by serines, site-directed mutagenesis was performed with the oligonucleotide pairs 5'-GAGTTTGCCACAATTGTCGATGTG-3', 5' -AGATGGGCTAAAGCCACTCCACTG-3', 5'-CAGTGGAGTGGCTTTAGCCCATCT-3' and 5'-CCACCGGTGCTAAGTCTGTCCTGTTCTC-3'. A HindIII/BamHI cDNA restriction fragment of EFP1 was subcloned into the pcDNA6/Myc-His C vector (Invitrogen) to generate the construct EFP1-Myc. For the N-terminally His-tagged Par-4 cDNA construct (denoted His ${ }_{6}-\mathrm{Par}-4$ ), the EcoRI fragment of the Par-4 cDNA from pCB6 $6^{+}-\mathrm{Par}-4^{29}$ was subcloned into the pPROEX ${ }^{\mathrm{TM}} \mathrm{HTa}$ expression vector (Invitrogen $\mathrm{GmbH}$, Karlsruhe, Germany). The construction of FLAG-Par-4 and the leucine zipper mutants FLAG-Par-4 L3A and His $_{6}$-Par-4 L3A, carrying exchanges of leucines 295, 316 , and 330 to alanines, has been described previously. ${ }^{21,23}$ 


\section{RNA expression analysis by Northern blotting and RT-PCR}

A blotting membrane containing $\sim 2 \mu \mathrm{g}$ of poly(A) ${ }^{+} \mathrm{RNA}$ per lane from eight different rat tissues (Clontech) was used for Northern analysis. The filter was hybridized with a $\left[\alpha-{ }^{32} \mathrm{P}\right] \mathrm{dCTP}-$ labeled EFP1 cDNA-fragment and with a $\left[\alpha-{ }^{32} \mathrm{P}\right] \mathrm{dCTP}-$ labeled glyceraldehyde-3-phosphate dehydrogenase (GAPDH) probe. Densiometric analysis of EFP1 and GAPDH expression was performed using ImageJ software (National Institutes of Health, Bethesda, MD, USA). For reverse transcriptase-polymerase chain reaction (RT-PCR) analysis, total RNA from various cultured cell lines (REF52.2, PC3, C6, E14-2, TZ102, TZ103, TZ107, N2A and NIH3T3) was prepared using the TRIzol ${ }^{\circledR}$ reagent (Invitrogen) according to the manufacturer's recommendations. In some experiments, the cells were treated with DMSO (0.72\% for $24 \mathrm{~h})$, ionomycin (10 $\mu \mathrm{M}$ for $24 \mathrm{~h})$, tunicamycin ( $3 \mu \mathrm{M}$ for $24 \mathrm{~h})$, thapsigargin ( $4 \mu \mathrm{M}$ for $24 \mathrm{~h})$, brefeldin A $(35.7 \mu \mathrm{M}$ for $24 \mathrm{~h}), \mathrm{H}_{2} \mathrm{O}_{2}(1 \mathrm{mM}$ for $24 \mathrm{~h})$ or staurosporine ( $10 \mathrm{nM}$ for $16 \mathrm{~h}$ ) prior to preparation of RNA. RNA $(2.5 \mu \mathrm{g})$ was reverse transcribed by SuperScript II ${ }^{\circledR}$ Reverse Transcriptase (Invitrogen) using a 17mer oligo-dT primer. Expression of EFP1 was analyzed by PCR with oligonucleotides 5'-CCCCATGAGTGCTGTTTACATT-3' and 5'-CGTACTGTTCCTAGGTAATC-3' amplifying a $219 \mathrm{bp}$ product of EFP1 and expression of GRP78/BiP with oligonucleotides 5'-GAAAGGATGGTTAATGATGCTGAG$3^{\prime}$ and 5'-GTCTTCAATGTCCGCATCCTG-3' amplifying a 231 bp GRP78/BiP product. As internal control, a 309 bp GAPDH product was amplified with the oligonucleotides 5'-CGGTGTGAACGGATTTGGCCGTAT-3' and 5'AGCCTTCTCCATGGTGGTGAAGAC-3'.

\section{Computer-assisted sequence analysis}

To analyze the EFP1 structure in detail, multiple sequence alignments were performed with the ClustalW program. ${ }^{30}$ For secondary structure predictions, the PredictProtein ${ }^{31}$ and the Paircoil programs ${ }^{32}$ were employed and the analyses of intracellular localization, posttranslational modifications, and signal peptide predictions were performed with the PSORT II, ${ }^{33}$ NetPhos, ${ }^{34}$ NetNGlyc, and SignalP programs. ${ }^{35}$

\section{Cell culture and transfection}

Rat embryo fibroblast line REF52.2, rat fetal brain cell line E14-2, human cervical carcinoma cell line HeLa, rat glioma cell line C6, rat PNET cell lines TZ102, TZ103 and TZ107 and green monkey kidney fibroblast cell line COS-1 were maintained in Dulbecco's modified Eagle medium (DMEM;
Sigma, Deisenhofen, Germany) supplemented with 10\% fetal calf serum (FCS; PAA Laboratories, Vienna, Austria). Human prostate cancer cell line PC3 was cultured in RPMI supplemented with $10 \%$ FCS. Mouse neuroblastoma cell line N2A was maintained in modified Eagle's medium (MEM; PromoCell, Heidelberg, Germany) supplemented with 10\% FCS, $1 \%$ glutamine and $1 \%$ nonessential amino acids (Sigma). Hamster ovarian cells (CHO) were grown in Nutrient Mixture F12 (Sigma) with 10\% FCS. Mouse immortal fibroblast cell line NIH3T3 was cultured in DMEM high glucose (Sigma) with 10\% FCS and 1\% glutamine. All cell culture media were supplemented with 100 units/ml penicillin and $100 \mu \mathrm{g} / \mathrm{ml}$ streptomycin. For transfection experiments, cells were seeded at 1000-3000 cells per $\mathrm{mm}^{2}$ and transiently transfected using the jetPEI transfection reagent (PolyPlus, Illkirch, France) according to the manufacturer's protocol. For the generation of stable EFP1-expressing cell lines, $\mathrm{CHO}$ cells were transfected with EFP1-GFP expression vector and selected in the presence of $1 \mu \mathrm{g} / \mathrm{ml}$ Geneticin (G418) (Life Technologies, Inc, Karlsruhe, Germany). EFP1-GFP expressing colonies were selected and further cultured. For the Par-4 siRNA experiments, cells were transiently transfected with 20 pmol of Cy3-labeled 21-nucleotide double-stranded RNA molecules (Perbio Science Germany, Bonn) using the Lipofectamine 2000 reagent (Invitrogen) according to manufacturer's protocol. The following Par4 siRNA oligonucleotides were employed: human Par-4 sense 5'-Cy3-GAUGCAAUUACACAACAG AUU-3' and human Par-4 antisense 3'-UUCUACGUUAAUGUGUUGUCU-Cy3-5'. ${ }^{36}$ As mismatch control, siRNA oligonucleotides against rat Par-4 were used: rat Par-4 sense 5'-Cy3-GAUGCUAUCACACAGCAGAUU-3' and rat Par-4 antisense 3'-UUCUACGAUAGUGUGUCGUCUCy3 $-5^{\prime} .48 \mathrm{~h}$ after transfection the cells were examined by fluorescence microscopy.

\section{Immunofluorescence analysis}

For immunofluorescence analysis, transfected cells were fixed with $3 \%$ paraformaldehyde and stained with the mouse monoclonal anti-c-Myc antibody 9E10 (1:200; Biomol $\mathrm{GmbH}$, Hamburg, Germany), the rabbit polyclonal antiPar-4 antibody (1:5000; Santa Cruz Biotechnology Inc.) and the rabbit polyclonal anti-PDI antibody (1:4000, Biomol). As secondary antibodies, $\mathrm{Cy} 3$-conjugated goat anti-mouse IgG, Alexa488-conjugated goat anti-rabbit IgG and Cy3-conjugated goat anti rabbit IgGs (1:1000; Dianova, Hamburg, Germany) were used. Nuclei were stained with 4,6-diamidino-2-phenylindole (DAPI). Cells were examined 
with an Axiophot fluorescence microscope (Carl Zeiss GmbH, Oberkochen, Germany) equipped with a CCD camera using filters optimized for double-label experiments and a $63 \times$ oil immersion objective. Confocal microscopy was performed with a Zeiss Axioplan fluorescence microscope coupled with a Zeiss LSM510. Images were processed with Adobe photoshop 7.0 software.

\section{Protein expression and purification}

His $_{6}$-Par-4, His 6 -Par-4 L3A, His 6 -EFP1 and His ${ }_{6}$-Amida were expressed in the E. coli strain BL21-CodonPlus DE3 (Stratagene). Bacteria were transformed with either expression vector and grown in LB-medium at $37^{\circ} \mathrm{C}$. Protein expression was induced in late $\log$ phase with $1 \mathrm{mM}$ isopropyl-1thio- $\beta$-D-galactopyranoside (IPTG) at $20-25^{\circ} \mathrm{C}$. Bacteria were harvested $5 \mathrm{~h}$ after induction and resuspended in

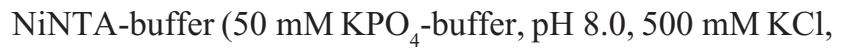
$10 \mathrm{mM}$ imidazole, $5 \mathrm{mM}$ 2-mercaptoethanol, 0.2\% Tween, $1 \mathrm{mM}$ PMSF). The cells were lysed with $1 \mathrm{mg} / \mathrm{ml}$ lysozyme for $15 \mathrm{~min}$ at $4{ }^{\circ} \mathrm{C}$ in the presence of 2 units $/ \mathrm{ml}$ Benzonase (Merck Biosciences GmbH, Schwalbach, Germany). Lysates were solubilized by ultrasonic disruption and cleared from cell debries by centrifugation. Recombinant proteins were purified using a NiNTA-matrix (IBA GmbH, Göttingen, Germany) essentially according to the manufacturer's instructions. The samples were further purified on a NAP-5 column (Amersham), eluted in low salt buffer (20 mM Tris $\mathrm{pH} 7.0,20 \mathrm{mM} \mathrm{KCl}$ ) and concentrated on a Centricon Centrifugal Filter Unit with Ultracel YM-10 membrane (Millipore GmbH, Schwalbach, Germany).

\section{SDS-PAGE, Western Blot analysis and dot blot immunoassay}

Cells were lysed in isotonic lysis buffer (10 mM NaPO4, $\mathrm{pH}$ 8.0, $140 \mathrm{mM} \mathrm{NaCl}, 3 \mathrm{mM} \mathrm{MgCl}$, $1 \mathrm{mM}$ dithiothreitol, $0.5 \%$ Nonidet-P40 and $50 \mu \mathrm{M}$ leupeptin). The cell lysates were subjected to SDS-PAGE according to standard procedures and electrophoretically transferred onto nitrocellulose membranes (Schleicher and Schuell GmbH, Dassel, Germany). The membranes were stained either with the mouse monoclonal anti-GFP antibody (1:2000, Clontech), the mouse monoclonal anti-FLAG M2 antibody (1:5000, Invitrogen), the rabbit polyclonal (1:5000) and mouse monoclonal (1:2000) antiPar-4 antibodies (Santa Cruz Biotechnology Inc., Heidelberg, Germany) or the mouse monoclonal anti- $\alpha$-Tubulin antibody DM 1A (1:5000, Sigma). Bound antibodies were detected with peroxidase-conjugated secondary goat antimouse or goat anti-rabbit IgGs (1:5000, Dianova) using a SuperSignal ${ }^{\circledR}$ West Pico chemoluminescence detection kit (Perbio Science GmbH, Bonn, Germany). For dot blots, 10-250 ng of purified recombinant $\mathrm{His}_{6}$-Amida protein, $\mathrm{His}_{6}$ EFP1 protein and bovine serum albumine (BSA) were spotted onto nitrocellulose membranes (Schleicher and Schuell). After blocking, membranes were incubated with $3 \mu \mathrm{g} / \mathrm{ml}$ of purified recombinant $\mathrm{His}_{6}$-Par-4 or $\mathrm{His}_{6}$-Par-4 L3A protein for $2 \mathrm{~h}$ and further processed as described above.

\section{Insulin disulfide reduction assay}

The assay was performed essentially as described by Holmgren $^{37}$ in quartz cuvettes in a final volume of $120 \mu 1$. Briefly, the incubation mixtures contained 3.9 $\mu \mathrm{M}$ of purified recombinant $\mathrm{His}_{6}$-EFP1 protein, $\mathrm{His}_{6}$-EFP $^{\mathrm{THX}}{ }^{\mathrm{TH}}$ protein or recombinant thioredoxin protein from $E$. coli (Sigma), $0.1 \mathrm{M}$ potassium phosphate (pH 7.0), 2 mM EDTA and $0.13 \mathrm{mM}$ of bovine insulin (Sigma). The reaction was started by adding $0.33 \mathrm{mM}$ dithiothreitol to the mixture. The rate of insulin reduction was measured spectrophotometrically at $650 \mathrm{~nm}$ for $100 \mathrm{~min}$ as turbidity formation from the precipitation of the insoluble free B chain.

\section{In vitro translation and pulldown assay}

In vitro translated $\left[{ }^{35} \mathrm{~S}\right]$-methionine labeled EFP1 was generated with the TNT-coupled reticulocyte lysate system (Promega GmbH, Mannheim, Germany) using the EFP1Myc vector as template according to the manufacturer's protocol. The labeled EFP1 protein was incubated for $30 \mathrm{~min}$ at $4{ }^{\circ} \mathrm{C}$ with NiNTA beads in the absence or in the presence of purified $\mathrm{His}_{6}$-Par-4 wt or $\mathrm{His}_{6}-\mathrm{Par}-4 \mathrm{~L} 3 \mathrm{~A}$, respectively. After washing with pull-down buffer (50 mM KPO-buffer $\mathrm{pH} 7.0,5 \mathrm{mM} \mathrm{MgCl}, 100 \mathrm{mM} \mathrm{KCl}, 10 \mathrm{mM}$ imidazole, $5 \%$ glycerol, $0.2 \%$ Tween, $1 \mathrm{mM}$ PMSF), the bound proteins were eluted from the NiNTA beads with pull-down buffer containing $500 \mathrm{mM} \mathrm{KCl}$. The eluted proteins were separated by SDS-PAGE and analyzed by autoradiography.

\section{Immunoprecipitation and Endoglycosidase $\mathrm{H}$ treatment}

CHO cells stably expressing EFP1-GFP were transfected with FLAG-Par-4 or FLAG-vector alone as control. $24 \mathrm{~h}$ post-transfection, the cells were metabolically labeled with $250 \mu \mathrm{Ci}$ of $\left[{ }^{35} \mathrm{~S}\right] \mathrm{methionine}(10 \mathrm{mCi} / \mathrm{ml}$, Amersham $)$ per $9 \mathrm{~cm}$-culture dish in methionine-free DMEM for 2 h. After labeling, the cells were washed with ice-cold phosphate-buffered saline (PBS) and lysed in isotonic lysis buffer. The lysates were subjected to immunoprecipitation with the rabbit polyclonal anti-GFP antibody (Clontech). 
The antigen-antibody complexes were adsorbed to Protein A-Sepharose (Sigma), separated on 10\% SDS-PAGE and further analyzed by autoradiography. For Endoglycosidase $\mathrm{H}$ (EndoH) experiments, HeLa cells were transfected with EFP1-GFP. $24 \mathrm{~h}$ after transfection cells were lysed in isotonic lysis buffer and the cell lysates subjected to immunoprecipitation as described above. The antigen-antibody-sepharose complex was treated with $1 \mathrm{x}$ denaturation buffer $\left(0.5 \%\right.$ SDS, $1 \% \beta$-mercaptoethanol) at $100{ }^{\circ} \mathrm{C}$ for 10 minutes. The sepharose beads were sedimented and the supernatants were treated with EndoH (NEB) at $37{ }^{\circ} \mathrm{C}$ for $1 \mathrm{~h}$ according to the manufacturer's protocol. The samples were separated on $7.5 \%$ SDS-PAGE and further analyzed by Western blotting.

\section{Apoptosis assay}

CHO cells were co-transfected with FLAG-Par-4, EFP1-GFP and EFP $1^{\text {THX- }}-$ GFP or with empty FLAG or GFP vectors as controls. $48 \mathrm{~h}$ post-transfection, the cells were fixed with paraformaldehyde, stained with the mouse monoclonal antiFLAG M2 antibody and with DAPI to visualize nuclei. For Par-4 knockdown experiments, HeLa cells were transfected with either human Par-4 siRNA or as mismatch control with rat Par-4 siRNA oligonucleotides. After transfection, the cells were treated either with ionomycin $(10 \mu \mathrm{M}$ for $24 \mathrm{~h})$, thapsigargin ( $2 \mu \mathrm{M}$ for $24 \mathrm{~h})$, tunicamycin ( $3 \mu \mathrm{M}$ for $24 \mathrm{~h}$ ), etoposide ( $17 \mathrm{mM}$ for $24 \mathrm{~h}$ ), staurosporine ( $200 \mathrm{nM}$ for $5 \mathrm{~h}$ ) or with DMSO as a control $(0.72 \%$ for $24 \mathrm{~h})$. After fixation with formaldehyde, the cells were stained for endogenous Par-4 with the rabbit polyclonal anti-Par-4 antibody (Santa Cruz Biotechnology Inc.) and with DAPI to visualize nuclei. The percentage of apoptotic cells that showed fragmented nuclei, condensed chromatin and membrane blebbing was detected among the transfected cells by fluorescence microscopy, counting 100 to 200 EFP1/Par-4 positive cells or 100 to 200 Par-4-knockdown cells versus Par-4 positive cells in the siRNA experiments in each experiment. Data were collected from five independent experiments, and the significance was determined in a one-tailed paired t-test for EFP1/Par-4 co-transfection experiments, and a two-tailed paired t-test for Par-4 siRNA transfection experiments.

\section{Results \\ Molecular cloning and sequence analysis of rat EFPI}

In an attempt to identify new interaction partners of Par-4, we screened a rat cDNA library derived from the PNET cell line TZ103 ${ }^{27,38}$ with full length Par-4 as bait. Seven positive clones were identified from histidine negative plates which were also positive for $\beta$-galactosidase expression. Sequence analysis revealed one clone with $100 \%$ identity to the rat cDNA XM220122.2, and BLAST analyses showed 94\% and $90 \%$ sequence identity with the murine and human TXNDC11 (thioredoxin domain containing 11) cDNA sequences, encoding the EFP1. ${ }^{26}$ Thus, our isolated cDNA clone comprising an open reading frame for 947 amino acids represented the rat ortholog of TXNDC11/EFP1.

Computer-assisted analysis of the EFP1 amino acid sequence (see Materials and methods) revealed that the predicted protein contains an $\mathrm{N}$-terminal ER signal sequence or signal anchor sequence (aa 54-83) but no ER retention signal, a putative transmembrane domain (aa 60-76, see Figure 1A), a putative NLS (aa 849-865), a leucine zipper motif (aa 834-855), an arginine rich sequence (aa 814-850), several putative phosphorylation sites for protein kinase $\mathrm{C}(\mathrm{PKC})$ and casein kinase 2 (CK2) as well as several N-glycosylation sites. The N-terminal portion of EFP1 is rich in glycine (35\%, distributed between aa 5-50), thus facilitating $\mathrm{N}$-myristoylation and a putative membrane localization of the protein. The presence of two thioredoxin domains (Figure 1A, a, aa 104-207 and a', aa 659-764), whereas the $\mathrm{C}$-terminal one contains a putative catalytically active CXXC motif $\left({ }_{787} \mathrm{CGFC}_{790}\right)$, classifies the protein as a novel member of the thioredoxin-related family. To gain more information about the domain structure of EFP1, the $\alpha$-helix/ $\beta$-strand composition was analyzed using the PredictProtein analysis program. Three variations of the thioredoxin fold alpha/beta pattern $\beta-\alpha-\beta-\alpha-\beta-\alpha-\beta-\beta-\alpha^{39,40}$ were identified, two of them spanning the two thioredoxin domains a and a', the other one adjacent to the first thioredoxin domain (b, Figure 1A).

\section{EFPI is expressed in various tissues and cell lines}

To analyze the expression of EFP1 at the transcriptional level, we hybridized a multiple tissue Northern blot with a probe corresponding to the EFP1 nucleotides 1583-2170. As depicted in Figure 1B (upper panel), in the majority of the tissues tested, a single EFP1 transcript with an estimated size of $3.4 \mathrm{~kb}$ was detected. Expression of the EFP1 transcript was highest in liver (Figure 1B, lane 5), less prominent in heart, brain, lung and kidney (Figure 1B, lanes 1, 2, 4 and 7) and rather low in spleen and testis (Figure 1B, lanes 3 and 8). No EFP1 transcript could be detected in skeletal muscle (Figure 1B, lane 6). The housekeeping gene 
A

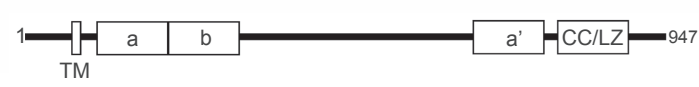

B

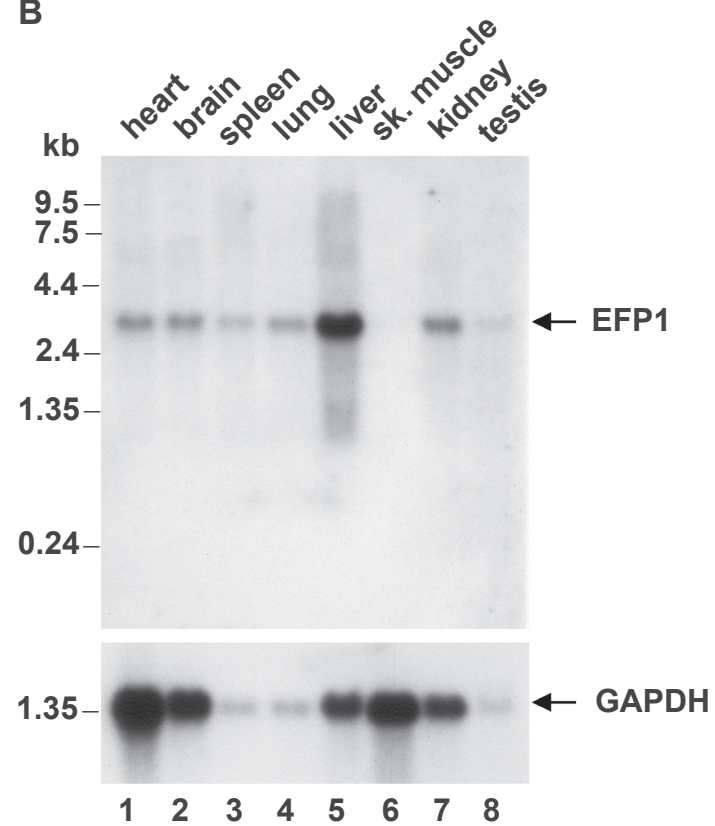

C

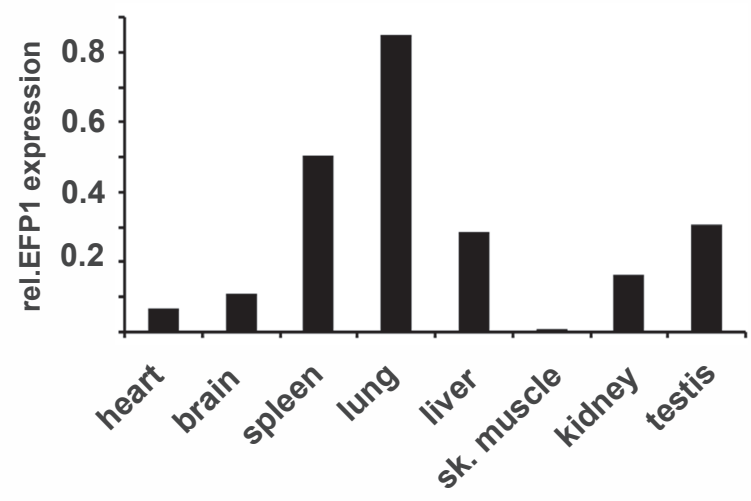

D

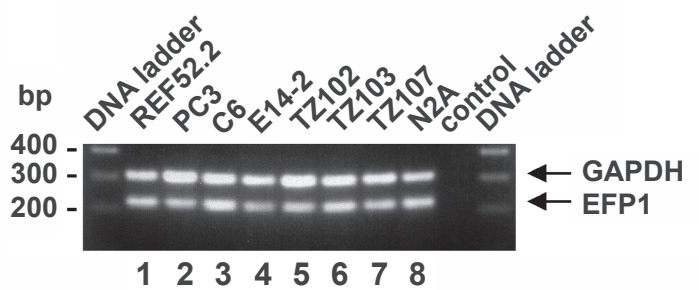

Figure I A) Schematic representation of the EFPI protein domain structure. Full length rat EFPI (947 residues) contains a transmembrane domain (TM), two thoredoxin domains (a and a'), a thioredoxin-like domain (b), a coiled coil domain (CC) and a leucine zipper motive (LZ). B) Expression of rat EFPI in different tissues and cell lines. A multiple tissue Northern blot (Clontech) containing $2 \mu \mathrm{g}$ of poly $(\mathrm{A})^{+}$RNA per lane from eight different rat tissues as indicated was probed with a cDNA fragment corresponding to rat EFPI nucleotides 1583-2I70 (upper panel). The same membrane was reprobed with a ${ }^{32} \mathrm{P}$-labeled full-length GAPDH cDNA probe to quantify the EFPI RNA expression relative to GAPDH expression (lower panel). C) Densiometric analysis of EFPI expression normalized to GAPDH in multiple tissues. The densiometric analysis of the EFPI and GAPDH bands detected after Northern blot analysis in (B) was performed using Image Software. D) Expression of EFPI and GAPDH in various cell lines analyzed by RT-PCR. cDNA from cell lines REF52.2, PC3, C6, EI4-2,TZI02, TZI03,TZI07 and N2A (lanes I-8) was amplified by PCR using conditions described under Experimental procedures. As internal standard, the house keeping gene GAPDH was amplified in the same PCR reactions.

Abbreviations: EFPI, EF-hand binding protein I; GAPDH, glyceraldehyde-3-phosphate dehydrogenase; RT-PCR, reverse transcriptase-polymerase chain reaction.

glyceraldehyde-3-phosphate dehydrogenase (GAPDH) was employed as reference (Figure 1B, lower panel).

Since the mRNA expression of GAPDH was quite variable between tissues, we performed a densiometric analysis of EFP1 and GAPDH bands detected after Northern blot analysis in Figure 1B using ImageJ software. The graph bar in Figure 1C shows the expression of EFP1 normalized to GAPDH. The densiometric analysis revealed that the relative expression level of EFP1 was highest in lung and spleen, less prominent in testis, liver and kidney and rather low in brain and heart. EFP1 expression was not detected in skeletal muscle.

In order to investigate the expression of EFP1 in transformed versus nontransformed cell lines, we performed semi-quantitative RT-PCR analysis with GAPDH as internal standard. We assayed the overall expression of EFP1 in nontransformed rat cells (REF52.2 fibroblasts, E14-2 fetal brain cells) and in transformed human prostate cancer cells (PC3), rat glioma cells (C6), rat PNET cell lines (TZ102, TZ103 and TZ107), and mouse neuroblastoma cells (N2A). As shown in Figure 1D (lanes 1-8), all cell lines tested displayed the same expression level of EFP1 comparable to the uniform expression of the housekeeping gene GAPDH. These data suggested that the expression of EFP1 is not altered in the examined nontransformed and tumor cell lines.

\section{Rat EFPI is localized in the ER}

To analyze the subcellular localization of rat EFP1 in mammalian cells, we examined C-terminally GFP- or Myc-tagged fusion proteins EFP1-GFP (Figure 2A, a-c) or EFP1-Myc (Figure 2A, d-f) in REF52.2 cells $24 \mathrm{~h}$ after transfection by confocal fluorescence microscopy. Since an ER localization has previously been described for the human ortholog of EFP $1,{ }^{26}$ we employed in our studies an anti-PDI 
A
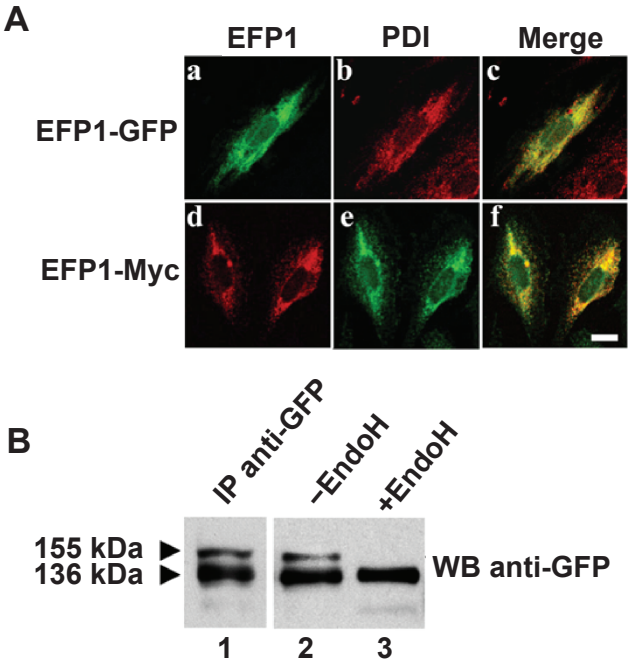

E

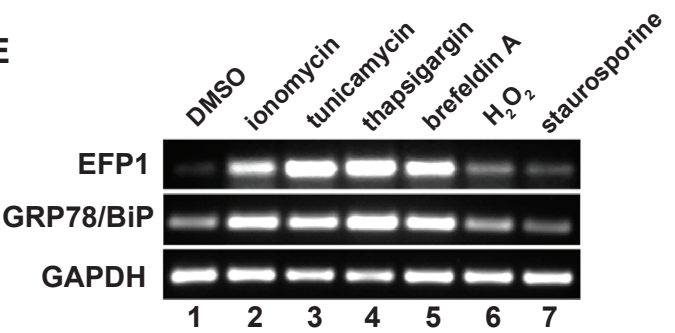

C
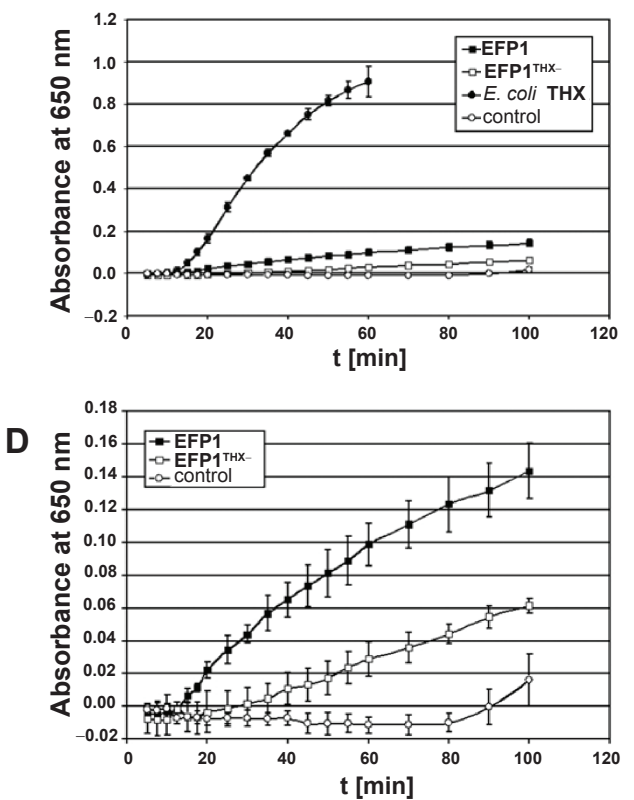

Figure 2 A) Subcellular localization of EFPI in REF52.2 cells. REF52.2 cells were transiently transfected with expression plasmids encoding either EFPI-GFP (a) or EFPI-Myc (d). $24 \mathrm{~h}$ post-transfection, the cells were fixed with paraformaldehyde and stained for indirect immunofluorescence microscopy with the mouse monoclonal anti-c-Myc antibody (d), with the rabbit polyclonal anti-PDI antibody (b and e) and with Cy3-labeled secondary antibodies. The yellow signals in the merged images (c and $f$ ) indicate colocalization. Scale bar, $20 \mu \mathrm{m}$. B) N-glycosylation analysis of EFPI. HeLa cells were transfected with the EFPI-GFP expression vector and 24 h post-transfection, cell lysates were prepared and subjected to immunoprecipitation with the polyclonal anti-GFP antibody (lane I). The immunoprecipitated EFPI-GFP proteins were further treated with EndoH (lane 3) or left untreated as control (lane 2). Arrows indicate the positions of the $136 \mathrm{kDa}$ or the $155 \mathrm{kDa}$ EFPI-GFP protein. Note that after EndoH treatment the $155 \mathrm{kDa}$ EFPI-GFP protein band is no longer detectable. C) EFPI catalyzes the reduction of insulin. $3.9 \mu \mathrm{M}$ recombinant affinity purified His ${ }_{6}$ EFPI protein (black squares) and $\mathrm{His}_{6}-\mathrm{EFPI}^{\mathrm{THX}}$-protein (white squares) were employed in a quantitative insulin disulfide reduction assay containing $0.13 \mathrm{mM}$ bovine insulin and $0.33 \mathrm{mM}$ dithiothreitol in a final volume of I $20 \mu \mathrm{l}$. Recombinant purified thioredoxin from E coli was included as positive control (black circles) and a sample containing only dithiothreitol served as negative control (white circles). The rate of insulin reduction was measure spectrophotometrically at $650 \mathrm{~nm}$ as turbidity formation from the precipitation of free insulin B chain plotted against time. D) The same experiment as shown in (C) without the data obtained from the $E$ coli thioredoxin experiments and plotted with an altered $y$-axis scale. The results are presented as mean values from three independent experiments. E) Analysis of EFPI expression upon ER stress. NIH3T3 cells were treated with the indicated drugs (lanes 2-7) and with DMSO as control (lane I). 16-24 h after treatment, the cells were harvested and the expression of EFPI was analyzed by RT-PCR (upper panel). The expression of GAPDH (lower panel) and the expression of GRP78/BiP (middle panel) served as internal controls. Note that the expression of EFPI is clearly upregulated in NIH3T3 cells treated with ER stress-inducing agents.

Abbreviations: GFP, green fluorescence protein; PDI, protein disulphide isomerase; EndoH, endoglycosidase H; DMSO, dimethyl sulfoxide; GRP78/Bip, glucose-regulated protein 78/immunoglobulin binding protein; THX, thioredoxin.

antibody as marker for ER staining (Figure 2A, b and e). Both EFP1-GFP and EFP1-Myc (Figure 2A, a and d) were localized in the ER of the transfected cells and showed a clear colocalization with PDI (Figure 2A, b and e and merged images in $\mathrm{c}$ and $\mathrm{f}$ ).

\section{EFPI is posttranslationally modified by $\mathrm{N}$-glycosylation}

When we transfected the EFP1-GFP construct into CHO cells and analyzed the expression of EFP1-GFP by Western blot analysis with a monoclonal anti-GFP antibody, we detected two protein bands of approximately 136 and $155 \mathrm{kDa}$. Detailed sequence analysis of EFP1 revealed that the protein comprises several potential N-glycosylation sites.
Since glycosylation can significantly alter the apparent molecular weight of a protein in SDS-PAGE analysis, we examined if glycosylation of EFP1 might be responsible for the two EFP1 protein variants observed by Western blot analysis. Hence, we subjected cell extracts from EFP1-GFP transfected HeLa cells to immunoprecipitation with a polyclonal anti-GFP antibody and treated the immunoprecipitated proteins with endoglycosidase $\mathrm{H}$ (EndoH). EndoH treatment specifically removes high-mannose type N-glycans which are typical for ER-resident proteins, but does not hydrolyze complex glycans that are generated by posttranslational modification in the Golgi apparatus. As control, we employed immunoprecipitated protein samples without EndoH treatment. The samples were analyzed by Western blotting with the 
monoclonal anti-GFP antibody. Immunoprecipitation of EFP1-GFP with the anti-GFP antibody specifically precipitated two proteins of about 136 and $155 \mathrm{kDa}$ representing the two EFP1-GFP variants (Figure 2B, lane 1). When the immunoprecipitated proteins were treated with Endo $H$, the $136 \mathrm{kDa}$ EFP1-GFP protein band was detected, whereas the $155 \mathrm{kDa}$ EFP1-GFP protein band was no longer visible (Figure 2B, lane 3). Thus, our results clearly demonstrated that EFP1 is a high mannose type-glycoprotein.

\section{EFPI possesses enzymatic activity}

Since the C-terminal thioredoxin domain (THX II) of EFP1 contains an active site CXXC motif, typical for members of the thioredoxin (Trx) family, we next wanted to examine whether this thioredoxin domain of EFP1 is functional. For this purpose, we employed recombinant affinity purified $\mathrm{His}_{6}{ }_{-}^{-}$ tagged EFP1 wt protein (designated $\mathrm{His}_{6}-\mathrm{EFP} 1$ ) and a $\mathrm{His}_{6}-$ EFP $1^{\mathrm{THX}-}$ mutant carrying cysteine to serine substitutions in the active site CXXC motif in an insulin disulfide reduction assay. The quantitative assay was developed to measure the rate of insulin reduction spectrophotometrically at $650 \mathrm{~nm}$ as turbidity formation from the precipitation of the free insulin B chain. ${ }^{37}$ As control, we included recombinant purified thioredoxin from $E$. coli (denoted $E$. coli THX) in our experiment, which has dithiol-disulfide-oxidoreductase activity and catalyzes the reduction of insulin by dithiothreitol. The recombinant $\mathrm{His}_{6}$-EFP1 wt protein (Figures $2 \mathrm{C}$ and D, black squares) indeed catalyzes the reduction of insulin disulfides, although the kinetic of the reaction showed a markedly slower rate compared to THX from $E$. coli (Figures $2 \mathrm{C}$ and D, black circles). That the catalytic activity of EFP1 was dependent on the putative active site motif CXXC in the THX II-domain of EFP1 was demonstrated with the recombinant $\mathrm{His}_{6}-\mathrm{EFP}^{\mathrm{THX}-}$ mutant (Figures 2C and D, white squares) which showed reduced insulin disulfide reduction activity compared to the His $_{6}$-EFP1 wt protein (Figures 2C and D, black squares). However, the catalytic activity of the $\mathrm{His}_{6}-\mathrm{EFP} 1^{\mathrm{THX}-}$ mutant was not completely abolished compared to the control sample containing only dithiothreitol (Figures 2C and D, white circles).

\section{Upregulation of EFPI in NIH3T3 cells after ER stress}

Because EFP1 is an ER-resident protein that possesses catalytic activity comparable to thioredoxin and structural similarities to members of the PDI family of ER chaperones, we speculated that EFP1 might be involved in the ER stress response. To test this hypothesis, we treated NIH3T3 cells with the ER stress-inducing agents tunicamycin and brefeldin A, with the $\mathrm{Ca}^{2+}$ stress inducing agents thapsigargin and ionomycin, the latter also inducing ER stress. Furthermore, the cells were treated with the oxidative stress inductor $\mathrm{H}_{2} \mathrm{O}_{2}$, with staurosporine (STS), which induces apoptosis independently of ER stress, and with DMSO as control. The expression of EFP1 was examined by RT-PCR analysis (Figure 2E, upper panel) with GAPDH as internal control (Figure 2E, lower panel). We also determined the expression of the ER stress marker GRP78/BiP (Figure 2E, middle panel). Treatment of NIH3T3 cells with either ionomycin, tunicamycin, thapsigargin, or brefeldin A (Figure 2E, upper panel, lanes 2-5), clearly resulted in the upregulation of EFP1. In analogy to these findings, the expression of the ER stress marker GRP78/BiP was also upregulated after ionomycin, tunicamycin, thapsigargin and brefeldin A treatment (Figure 2E, middle panel, lanes 2-5). In contrast, neither EFP1 nor GRP78/BiP expression was affected after induction of oxidative stress by $\mathrm{H}_{2} \mathrm{O}_{2}$ (Figure 2E, lane 6) or after ER-independent induction of apoptosis by staurosporine (Figure 2E, lane 7), thus demonstrating that the observed upregulation of EFP1 represents a specific ER stress-response.

\section{EFPI interacts with Par-4}

Since the rat EFP1 cDNA was originally isolated in a yeast two-hybrid analysis with Par-4 as bait, we wanted to confirm the interaction of full length EFP1 with Par-4, both in vitro and in vivo. For the in vitro binding assays, Par-4 was expressed as $\mathrm{His}_{6}$-tagged fusion protein in E. coli, immobilized on NiNTA beads and incubated with ${ }^{35}$ S-methioninelabeled in vitro-translated EFP1 protein. After stringent washing, the bound proteins were eluted from the NiNTAmatrix and analyzed by SDS-PAGE. In order to test whether the complex formation of EFP1 and Par-4 was dependent on a functional leucine zipper of Par-4, we also employed a His $_{6}$-tagged leucine-zipper point mutant of Par-4 (denoted His $_{6}$-Par-4 L3A), which contained three exchanges of leucine residues to alanines in the coiled coil interface. As shown in Figure 3A, EFP1 was pulled down together with $\mathrm{His}_{6}$-Par-4 wt (Figure 3A, lane 3) and with $\mathrm{His}_{6}-\mathrm{Par}-4 \mathrm{~L} 3 \mathrm{~A}$ (Figure 3A, lane 4), but not with NiNTA beads alone which served as negative control (Figure 3A, lane 2). Since equal amounts of EFP1 were eluted from the NiNTA-Par-4 wt and the NiNTA-Par-4 L3A matrixes, these results suggested that the interaction between EFP1 and Par-4 is not mediated by the leucine zipper of Par-4.

To further corroborate these findings, we performed dot-blot experiments with EFP1, Par-4 wt and Par-4 L3A. 


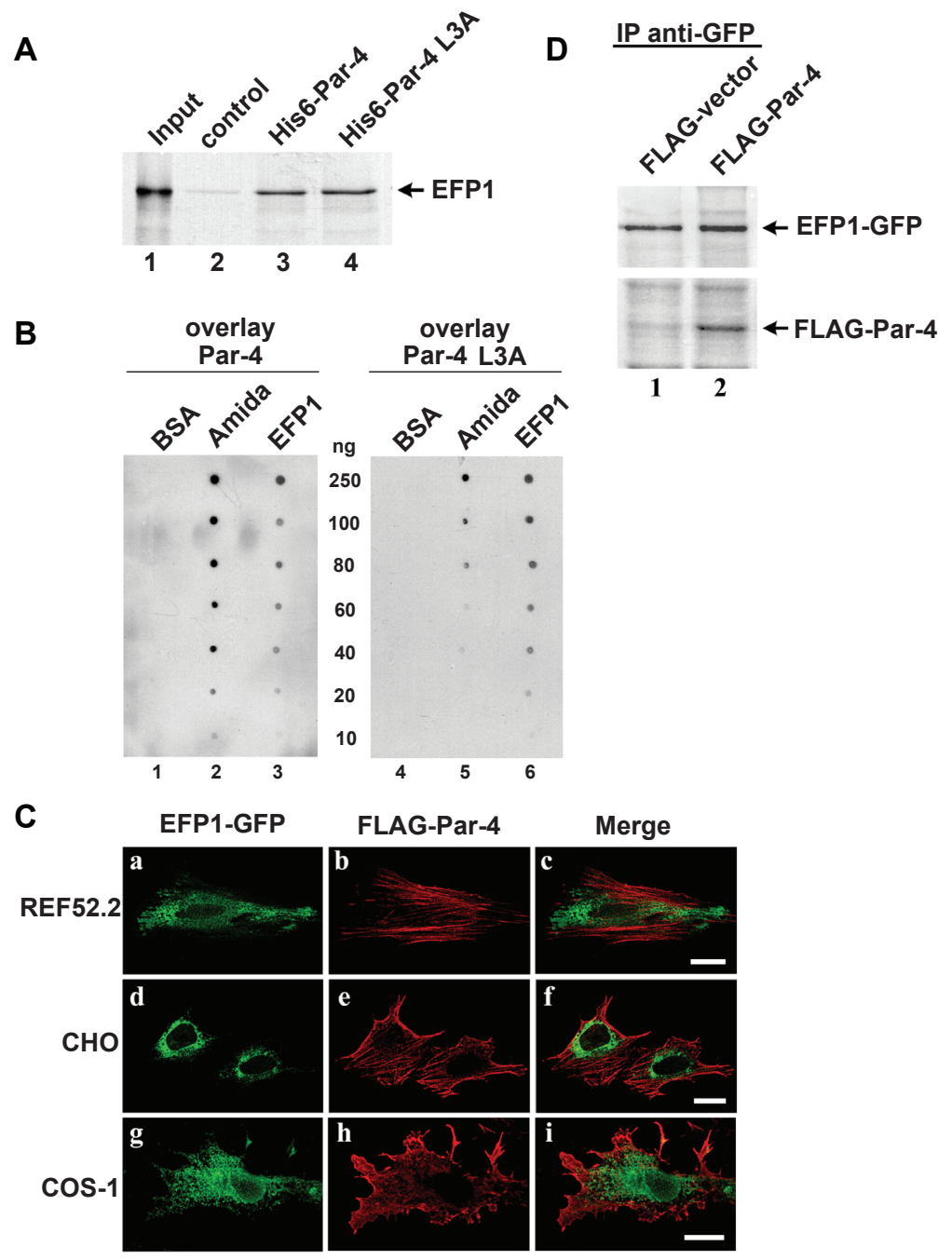

Figure 3 Analysis of EFPI and Par-4 interaction. A) Pull-down experiment using recombinant His - -Par-4 fusion protein (lane 3) and His - Par-4 L3A fusion protein (lane 4) immobilized on a NiNTA matrix and in-vitro translated ${ }^{35} \mathrm{~S}$-methionine-labeled EFPI. NiNTA beads alone served as negative control (lane 2). Samples were further processed and analyzed by SDS-PAGE and subsequent fluorography. The input (lane I) represents I/IO of the material used in the binding reactions. B) Dot blot analysis of EFPI and Par-4. 10-250 ng of purified recombinant His -EFPI protein (lanes 3 and 6), His -Amida protein (positive control, lanes 2 and 5) and BSA (negative control, lanes I and 4) were spotted as dots on nitrocellulose membranes. The membranes were incubated with $3 \mu \mathrm{g} / \mathrm{ml}$ of purified recombinant His ${ }_{6}$-Par- 4 wt protein (lanes $\mathrm{I}-3$ ) or purified recombinant $\mathrm{His}_{6}-\mathrm{Par}-4$ L3A mutant protein (lanes 4-6) and further processed as described for Western blot analysis with the monoclonal anti-Par-4 antibody. C) Coexpression of EFPI and Par-4. REF52.2, CHO and COS-I cells were cotransfected with EFPI-GFP and FLAG-Par-4 expression vectors. $24 \mathrm{~h}$ post-transfection, the cells were fixed and stained with the monoclonal anti-FLAG M2 antibody and with Cy3-labeled antibodies (b, c and h). No colocalization could be detected in the EFPI-GFP/FLAG-Par-4 coexpressing cells (merged images in c, f, and i). Scale bar, $20 \mu \mathrm{m}$. (D) Co-immunoprecipitation of EFPI and Par-4. CHO cells stably expressing an EFPI-GFP fusion protein were transfected either with FLAG-Par-4 (lane 2) or empty FLAG-vector as control (lane I). $24 \mathrm{~h}$ after transfection, the cells were metabolically labeled with [35 $]$ methionine and the cell lysates subjected to immunoprecipitation with the rabbit polyclonal anti-GFP antibody. The immunoprecipitated proteins were separated on a 7.5\% SDS-PAGE and analyzed by autoradiography. Arrows indicate the positions of FLAG-Par-4 and the EFPI-GFP fusion proteins.

Abbreviations: BSA, bovine serum albumin; NiNTA, nickel-nitrilotriacetic acid.

As positive control we included the novel Par-4 interaction partner Amida in our study, since it has been shown that complex formation between Par-4 and Amida requires a functional leucine-zipper of Par-4. ${ }^{21}$ Hence, purified recombinant EFP1 and Amida proteins were spotted as dots on nitrocellulose membranes. Bovine serum albumin (BSA) served as negative control (Figure 3B). Next, the membranes were incubated with purified recombinant Par-4 (Figure 3B, lanes 1-3) or Par-4 L3A (Figure 3B, lanes 4-6) and subsequently stained with an anti-Par-4 antibody. Par-4 wt was detected in a concentration-dependent manner on both the Amida and EFP1 spotted membranes (Figure 3B, lane 2 and 3) but no reaction with the BSA protein was observed (Figure 3B, lane 1). Interestingly, whereas the binding of Par-4 L3A to Amida was clearly reduced (Figure 3B, compare lanes 2 and 5), binding of Par-4 wt and Par-4 L3A to EFP1 was equally strong (Figure 3B, compare lanes 3 and 6 ). Thus, confirming our previous results that the 
interaction of Par-4 and EFP1 does not require the leucine zipper of Par-4.

To verify the interaction of EFP1 and Par-4 in vivo, cotransfection experiments with REF52.2, CHO and COS-1 cells were carried out with FLAG-Par-4 and EFP1-GFP. $24 \mathrm{~h}$ post-transfection, the cells were fixed, immunostained and analyzed by confocal fluorescence microscopy (Figure 3C). As expected, FLAG-Par-4 was clearly associated with actin filaments in REF52.2 cells and CHO cells (Figure 3C, b and e), whereas Par-4 was diffusely distributed in COS-1 cells (Figure 3C, h), which lack a well organized actin cytoskeleton. In contrast, the EFP1-GFP construct showed no association with stress fibers in the FLAG-Par-4/EFP1-GFP coexpressing cells, but rather displayed an ER specific fluorescence staining (Figure $3 \mathrm{C}$, a, $\mathrm{d}$ and $\mathrm{g}$ ).

We then performed co-immunoprecipitation experiments with CHO cells stably expressing an EFP1-GFP fusion protein and transfected the cells with either FLAG-Par-4 or empty FLAG-vector as control. $24 \mathrm{~h}$ after transfection, the cells were metabolically labeled with $\left[{ }^{35} \mathrm{~S}\right]$-methionine and the cell lysates were subjected to immunoprecipitation with the rabbit polyclonal anti-GFP antibody. A protein of about $48 \mathrm{kDa}$ representing the FLAG-Par-4 fusion protein was specifically coprecipitated with EFP1-GFP (Figure 3D, lane 2) which could not be detected in mock-transfected cells (Figure 3D, lane 1). In summary, our data provide strong evidence that EFP1 and Par-4 interact under physiological conditions, although no colocalization was observed by immunofluorescence analysis.

\section{Coexpression of EFPI and Par-4 leads to enhanced apoptosis}

Since we could show a physical interaction between EFP1 and the pro-apoptotic protein Par-4, we next asked whether EFP1 is also involved in Par-4-mediated apoptosis. To address this question, we co-transfected CHO cells with EFP1-GFP and FLAG-Par-4, fixed the cells $48 \mathrm{~h}$ post-transfection and stained the cells with the monoclonal anti-FLAG M2 antibody and with DAPI to visualize nuclei. Figure 4A (a-d) represents a cell co-transfected with EFP1-GFP and FLAG-Par-4 with normal morphology, whereas Figure 4A (e-h) represents a cell co-transfected with EFP1-GFP and FLAG-Par-4 with apoptotic morphology, ie, fragmented nuclei, condensed chromatin, and membrane blebbing.

In order to determine the percentage of apoptotic cells among the EFP1 and Par-4 co-expressing cells, we transfected CHO cells either with FLAG-Par-4, EFP1-GFP,

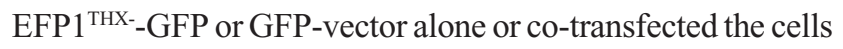

with FLAG-Par-4/EFP1-GFP or FLAG-Par-4/EFP1 ${ }^{\text {THX--GFP. }}$ $48 \mathrm{~h}$ post-transfection, the cells were fixed, immunostained with the monoclonal anti-FLAG M2 antibody and with DAPI to visualize nuclei and analyzed for morphological features of apoptosis. As shown in Figure 4B, the expression of the pro-apoptotic protein Par-4 alone resulted in induction of apoptosis of about $24 \%$ in CHO cells, whereas the expression of EFP1 leads to induction of apoptosis in about $10 \%$ of the transfected cells. Coexpression of EFP1 and Par-4 wt significantly increased the induction of apoptosis (about $30 \%$, $\mathrm{p}<0.05$ ), in contrast to $25 \%$ of apoptotic cells coexpressing Par-4 wt and mutant EFP1 ${ }^{\mathrm{THX}-}$. Thus, indicating that the catalytic activity of EFP1 is required for the Par-4/EFP1mediated apoptosis.

\section{siRNA oligonucleotide-mediated knockdown of Par-4 inhibits $\mathrm{Ca}^{2+}$ stress-mediated apoptosis}

Our findings demonstrate that Par-4 and EFP1 seem to play an important role in ER stress-mediated apoptosis. In order to corroborate these findings, we performed knockdown experiments in $\mathrm{HeLa}$ cells employing Cy3-labeled double stranded siRNA oligonucleotides against human Par-4. In order to show the specificity of the human Par-4 siRNA oligonucleotides, we transfected HeLa cells with 20 pmol human Par-4 siRNA oligonucleotides or with rat Par-4 siRNA oligonucleotides as a mismatch control. $48 \mathrm{~h}$ after transfection, the expression of endogenous Par-4 was analyzed by Western blotting or by confocal fluorescence microscopy with the polyclonal anti-Par-4 antibody. After transfection with the human Par-4 siRNA oligonucleotides, only a faint Par-4 protein band of about $40 \mathrm{kDa}$ was detected (Figure 5A, lane 2, upper panel) compared to the strong Par-4 protein band in control cells (Figure 5A, lane 1, upper panel). In order to verify that the different amounts of Par-4 detected in the samples were not due to unequal protein loading, immunostaining of the same membrane was performed with an $\alpha$-tubulin antibody (Figure 5A, lower panel). The specificity of the Cy3-labeled double stranded human Par-4 siRNA oligonucleotides was further confirmed by confocal fluorescence microscopy (Figure 5 B). In control cells, a strong fluorescence staining was detected with the polyclonal anti-Par-4 antibody (Figure 5B, a), in contrast to the human Par-4 siRNA oligonucleotide transfected cells (Figure 5B, b).

We next examined the effect of the siRNA oligonucleotide-mediated knockdown of endogenous Par-4 on ER 
A

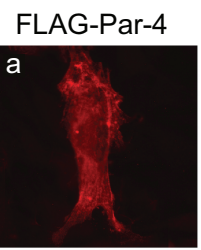

e

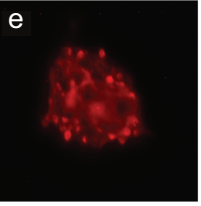

EFP1-GFP
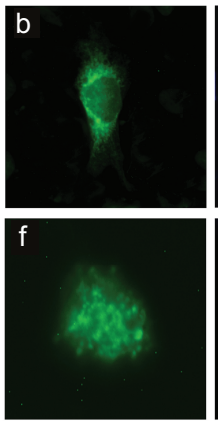

DAP1

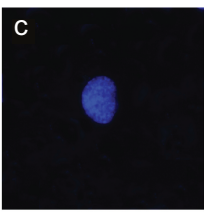

g
Merge

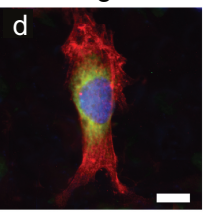

h

B

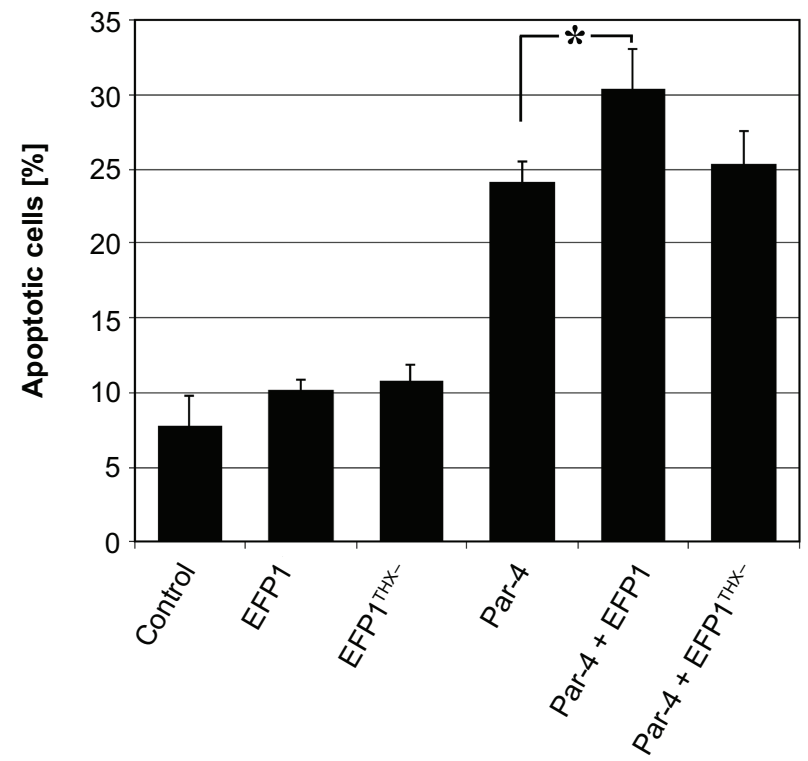

Figure 4 Apoptosis induction in CHO cells upon overexpression of EFPI-GFP and FLAG-Par-4. A) CHO cells were co-transfected with FLAG-Par-4 and EFPI-GFP. $48 \mathrm{~h}$ post-transfection, the cells were fixed, stained with the monoclonal anti-FLAG M2 antibody (a and e) and with DAPI (c and g) to visualize nuclei. (a-d) Representative cell co-transfected with FLAG-Par-4 and EFPI-GFP showing normal morphology and (e-h) representative cell co-transfected with FLAG-Par-4 and EFPI-GFP showing apoptotic morphology, ie, fragmented nuclei, condensed chromatin and membrane blebbing. Scale bar, $10 \mu \mathrm{m}$. B) Quantitative analysis of apoptosis induction upon coexpression of EFPI-GFP and FLAG-Par-4. CHO cells were transiently transfected either with EFPI-GFP, EFPI THX-GFP, FLAG-Par-4 or GFP-vector alone (control) or co-transfected with FLAG-Par-4/EFPI-GFP or FLAG-Par-4/EFPI THX-GFP. $48 \mathrm{~h}$ post-transfection, the cells were fixed, stained with the monoclonal anti-FLAG M2 antibody and with DAPI to visualize nuclei. The percentage of cells that showed apoptotic morphology (ie, fragmented nuclei, condensed chromatin and membrane blebbing; see also A, e-h) was determined among the transfected cells by fluorescence microscopy counting 100 to 200 positive cells in each experiment. The graph represents the mean values and standard errors from five independent experiments. Note that the difference in the percentage of apoptotic cells upon expression of Par-4 alone and coexpression of Par-4/EFPI (marked by an asterisk) is statistically significant $(\mathrm{p}=0.037)$.

Abbreviations: DAPI, 4, 6-diamidino-2-phenylindole; THX, thioredoxin.

stress-mediated apoptosis. Hence, we transfected HeLa cells with either human Par-4 siRNA or rat Par-4 siRNA as control, and treated the cells $48 \mathrm{~h}$ after transfection with agents inducing ER stress (ionomycin, thapsigargin, tunicamycin), with agents inducing ER stress independent apoptosis (etoposide and staurosporine) or with DMSO as control. Cells were fixed and stained with the rabbit polyclonal anti-Par-4 antibody and with DAPI to visualize nuclei. The percentage of apoptotic cells that showed fragmented nuclei, condensed chromatin and membrane blebbing was determined by fluorescence microscopy. As depicted in Figure 5C, treatment of HeLa cells with ionomycin induced apoptosis in about $17 \%$ of the cells (Figure 5C, white column), whereas the siRNA oligonucleotide-mediated knockdown of Par-4 resulted in $3 \%$ apoptotic cells (Figure 5C, black column). The percentage of apoptotic cells after treatment with thapsigargin of about $30 \%$ (Figure 5C, white column) was also significantly decreased to approximately $15 \%$ after siRNA-mediated Par-4 knockdown (Figure 5C, black column) $(\mathrm{p}<0.01$ for both agents in a two-tailed paired t-test). In contrast, no significant 
A

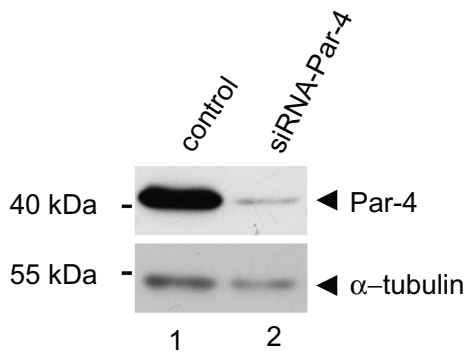

B

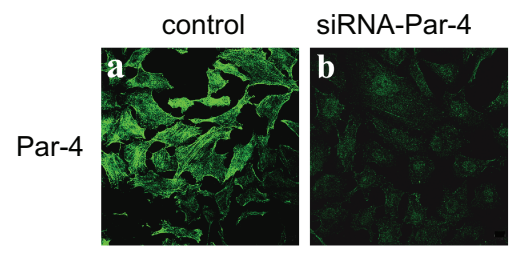

C

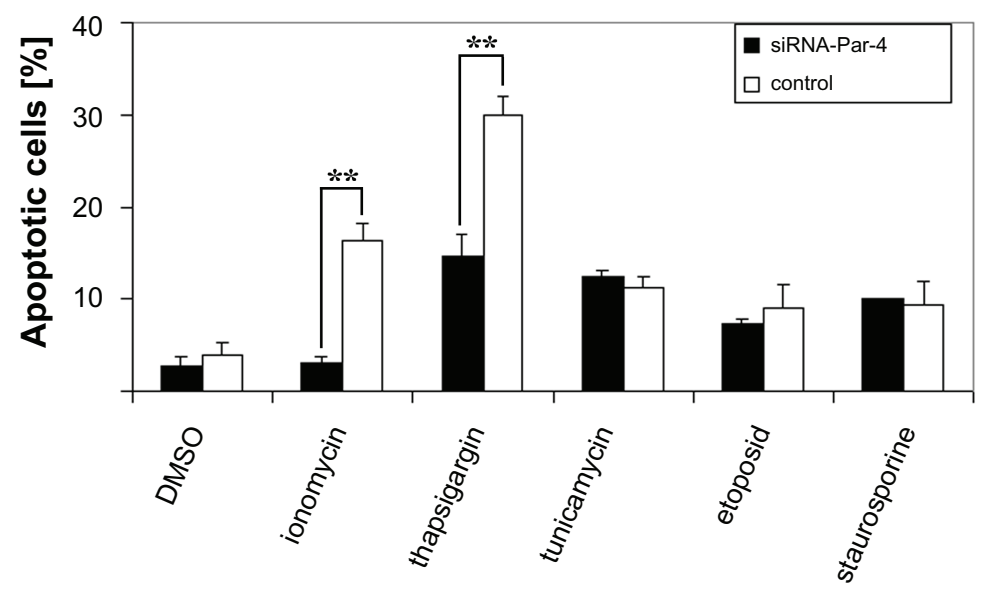

Figure 5 Effect of siRNA oligonucleotide-mediated knockdown of Par-4 on ER stress-mediated apoptosis. A) HeLa cells were transfected with either Cy3-labeled double stranded human Par-4 siRNA oligonucleotides (lane 2) or with rat Par-4 siRNA oligonucleotides as a mismatch control. (lane I). $48 \mathrm{~h}$ after transfection cell lysates were subjected to SDS-PAGE andWestern blot analysis with the rabbit polyclonal anti-Par-4 antibody (upper panel) or with the mouse monoclonal anti- $\alpha$-tubulin antibody as loading control (lower panel). B) HeLa cells were either transfected with 20 pmol of human Par-4 siRNA olgonucleotides (b) or with rat Par-4 siRNA oligonucleotides as a mismatch control (a). $48 \mathrm{~h}$ after transfection, cells were fixed with formaldehyde and stained with the rabbit polyclonal anti-Par- 4 antibody. Scale bar, $10 \mu \mathrm{m}$. C) HeLa cells were transfected with either human Par-4 siRNA oligonucleotides (black columns) or with rat Par-4 siRNA oligonucleotides as a mismatch control (white columns). $48 \mathrm{~h}$ after transfection cells were exposed to different apoptotic stimuli (ionomycin, thapsigargin, tunicamycin, etoposide or staurosporine) and as a control with DMSO. $16-24 \mathrm{~h}$ after treatment the cells were fixed and stained with the rabbit polyclonal anti-Par-4 antibody and with DAPI to visualize nuclei. The percentage of cells that showed apoptotic morphology (ie, fragmented nuclei, condensed chromatin and membrane blebbing) was determined by fluorescence microscopy counting 100 to 200 cells in each experiment exhibiting no Par-4 expression. The graph represents the mean values and standard errors from three independent experiments. Note that the decrease in the percentage of apoptotic cells after siRNA-mediated knockdown of Par-4 is statistically significant for ionomycin $(p=0.0043)$ and thapsigargin $(p=0.0019)($ marked by asterisks).

Abbreviations: DMSO, dimethyl sulfoxide; DAPI, 4, 6-diamidino-2-phenylindole.

decrease in apoptosis induction was observed after treatment of the cells with tunicamycin, etoposide or staurosporine after human Par-4 siRNA oligonucleotide transfection (Figure 5C, black columns), thus demonstrating that Par-4 might play an important role in $\mathrm{Ca}^{2+}$ stress-mediated apoptosis.

\section{Discussion}

In the present study, we report on the functional characterization of the rat ortholog of EFP1 and show that EFP1 is a novel interaction partner of the pro-apoptotic protein Par-4.

The rat EFP1 protein shares $79 \%$ similarity with the human EFP1 protein, with the highest sequence identity between amino acids 51-304 and 564-767. According to secondary structure predictions, the first conserved region of rat EFP1 comprises a putative thioredoxin domain with an inactive catalytic site and an adjacent region that is in good agreement with the thioredoxin fold. ${ }^{40}$ The second conserved region of the rat EFP1 protein also contains a putative thioredoxin domain but harbors a catalytically active CXXC motif $\left({ }_{787} \mathrm{CGFC}_{790}\right)$. The presence of two putative thioredoxin domains and the similarity in protein domain structure suggest that EFP1 is a member of the PDI family, a growing family of ER proteins that display isomerase, chaperone and calcium binding properties. ${ }^{41,42}$ The founding member, PDI, possesses the domain structure a-b-b'-a'-c, with a and a' representing the catalytically active thioredoxin domains, $b$ and b' the thioredoxin-like domains and $c$ the calcium binding domain. The b' domain has been implicated in substrate binding, whereas the $b$ domain appears to have a rather structural function. ${ }^{41}$ For full catalytic 
activity, all thioredoxin-like domains of PDI are required. ${ }^{43}$ In comparison, rat EFP1 contains the domain structure a-b$\mathrm{x}-\mathrm{a}$ '-CC/LZ, where CC/LZ represents the coiled coil and the leucine zipper domain and $\mathrm{x}$ an uncharacterized domain or a linker region (Figure 1A). Although no high sequence or structural similarities were identified between the $\mathrm{x}$ domain of rat EFP1 and the b' domain of PDI, the similar domain position suggests a possible function in substrate binding. Some of the PDI family members, like ERp29, possess a C-terminal alpha helical D domain that seems to be involved in substrate processing. ${ }^{42,44}$ However, the C-terminal coiled coil domain of rat EFP1 shows no sequence identity with the D-domain of the other PDI family members, arguing that this protein-protein interaction domain is unique for EFP1.

Analysis of the expression of EFP1 at the transcriptional level revealed a ubiquitous expression in various rat tissues, whereas no EFP1 transcript could be detected in rat skeletal muscle. Additionally, EFP1 is ubiquitously expressed in several tumor cell lines. Ectopic expression and immunostaining of rat EFP1-GFP and EFP1-Myc fusion proteins showed that the proteins reside in the ER as it has been demonstrated for the human ortholog. ${ }^{26}$ Unfortunately, no specific EFP1 antibodies are available to demonstrate the subcellular localization of the endogenous EFP1 protein. However, the presence of an ER signal anchor and the structural similarities with the members of the PDI protein family supports the ER distribution of the EFP1 protein. Furthermore, by employing an insulin disulfide reduction assay, we demonstrated that the purified recombinant EFP1 protein possesses catalytic activity and that the catalytic activity is dependent on the active site motif ${ }_{787} \mathrm{CGFC}_{790}$ located in the second thioredoxin domain of EFP1. The kinetics of the reaction with EFP1 showed a slower rate compared to recombinant purified thioredoxin from $E$. coli. Yet, it has to be taken into consideration that EFP1, like other thioredoxin-related proteins, might be involved in various redox regulations (eg, oxidation, isomerization). The catalytic activity of EFP1 was not completely abolished after substitution of the cysteine residues to serine residues in the active site CXXC motif; therefore it is likely that the catalytic activity of EFP1 only partially involves reduction activity. Our results are in good agreement with a previous report of Horibe and colleagues ${ }^{45}$ showing that the PDI family member PDIr retained over $50 \%$ of oxidative refolding activity towards $\alpha_{1}$-antitrypsin after exchanging all six catalytic site cysteine to serine residues.

We demonstrate in this study that EFP1 is a glycoprotein and that the glycosylation is of the high mannose type typical for ER-resident proteins. We do not know whether the highly glycosylated $155-\mathrm{kDa}$ form of EFP1 represents the mature active protein. It has been reported that the highly glycosylated 190-kDa form of the EFP1 interaction partner Duox corresponds to the mature active protein expressed at the plasma membrane and that the high mannose $180-\mathrm{kDa}$ form corresponds to the immature inactive protein which resides in the ER. ${ }^{26}$ Likewise, the ectopically expressed EFP1 protein could represent a less active protein compared to the endogenous EFP1 protein.

The expression of EFP1 was upregulated at the transcriptional level upon treatment of cells with agents that induce ER stress, but not by agents that induce oxidative stress or ER-independent apoptosis. Thus, EFP1 is specifically upregulated during ER stress, as it has been shown for a number of ER chaperones, including many members of the PDI family. ${ }^{46}$ Our findings suggest that EFP1 may function as a cell-protective enzyme and corroborate the assumption that EFP1 is a member of the PDI family. Unfortunately, no specific EFP1 antibodies are available to examine the upregulation of EFP1 after ER stress at the protein level. Clearly, this issue requires further investigation.

We have identified the rat ortholog of EFP1 in a yeast two-hybrid screen in search for new interaction partners of Par-4 that might serve as regulators or targets in Par-4mediated apoptosis. The interaction between Par- 4 and EFP1 was confirmed by pull-down experiments, dot blot analysis and co-immunoprecipitation experiments. These experiments corroborated a physical interaction of both proteins, although ectopically expressed EFP1-GFP and FLAG-Par-4 did not colocalize in the transfected cells. It has been demonstrated that many members of the PDI family and other ER proteins like calreticulin are not only localized in the ER but also in non-ER locations..$^{47} \mathrm{PDI}$ itself has been found in the nucleus ${ }^{48}$ as well as outside of the cell, either attached to the cell surface or in a secreted form. ${ }^{49}$ Overexpressed ERp57 was shown to be secreted from cultured cells ${ }^{50}$ and has been detected both in the cytosol ${ }^{51}$ and in the nucleus. ${ }^{48}$ The mechanism by which proteins of the PDI family can escape from the ER is unknown. It is therefore possible that by a similar mechanism, EFP1 can escape from the ER and interact with Par-4 in the cytoplasm to control the function of Par-4 after induction of ER stress.

Despite the well-established apoptotic action of Par-4, our understanding of molecular mechanisms controlling Par-4 function is limited. To date, only two regulatory mechanisms are known: the upregulation of Par-4 gene transcription, ${ }^{20,52}$ and the phosphorylation within the 59-residue core region, termed selective for apoptosis induction in cancer cells (SAC) 
domain $^{53}$ at the critical phosphorylation site T164/T155 (human and rat amino acid sequence, respectively). However, other regulatory mechanisms are likely to exist, since Par-4 is expressed in various tissues and cell lines without inducing apoptosis or sensitizing the cells for apoptosis. The novel Par-4 interaction partner EFP1 is a good candidate to regulate the function of Par-4 since i) coexpression of Par-4 and EFP1 in $\mathrm{CHO}$ cells resulted in enhanced Par-4-mediated apoptosis and ii) EFP1/Par-4-mediated apoptosis is dependent on the catalytic activity of EFP1. How EFP1 exerts its regulatory function on Par-4 remains elusive. It is tempting to speculate that EFP1 might function as a molecular chaperone or disulfide isomerase which can induce a conformational change of the Par-4 protein resulting in enhanced Par-4 activity. Indeed, the amino acid sequence of the rat Par-4 protein contains three cysteine residues at the position 82,103 , and 165 which are able to form disulfide bonds to stabilize the tertiary and/or quaternary structure of the protein.

As a potential regulator of Par-4, EFP1 provides a link between ER stress and Par-4-induced apoptosis. Par-4 has been suggested to play a significant role in ER stress-induced apoptosis in Alzheimer's disease, ${ }^{19}$ and deregulated expression of Par-4 has been implicated not only in Alzheimer's disease, ${ }^{54-57}$ but also in amyotrophic lateral sclerosis (ALS), Parkinson's disease and ischemic neuronal death. ${ }^{58-61}$ Furthermore, we could show in this study that Par-4 might play an important role in $\mathrm{Ca}^{2+}$ stress-mediated apoptosis, since the induction of apoptosis in the ionomycin and thapsigargin treated cells were significantly decreased after siRNA oligonucleotide-mediated knockdown of Par-4. That the siRNA oligonucleotide-mediated knockdown of Par-4 had no effect on apoptosis induction after treatment of the cells with the glycosylation inhibitor tunicamycin might be explained by the fact that EFP1 is a glycoprotein and thus, tunicamycin might hamper the function of EFP1 and Par-4.

Therefore, we propose a hypothetical model how EFP1 and Par-4 might determine the cell fate during ER stress (see Figure 6). In the presence of low Par-4 levels, EFP1 may act as a molecular chaperone or disulfide isomerase and contribute to restore the ER function (left part of Figure 6). If the ER stress response fails to restore the ER function, a disturbed calcium homeostasis (" $\mathrm{Ca}^{2+}$ stress") might be the consequence, leading to transcriptional upregulation of Par-4 as has been shown by Sells and colleagues and by Hsu and colleagues $^{20,52}$ and to the induction of apoptosis. In the presence of high Par-4 levels, EFP1 may function as an activator of Par-4, thus enhancing Par-4-mediated apoptosis (right part of Figure 6). According to our model, Par-4 itself is not fully

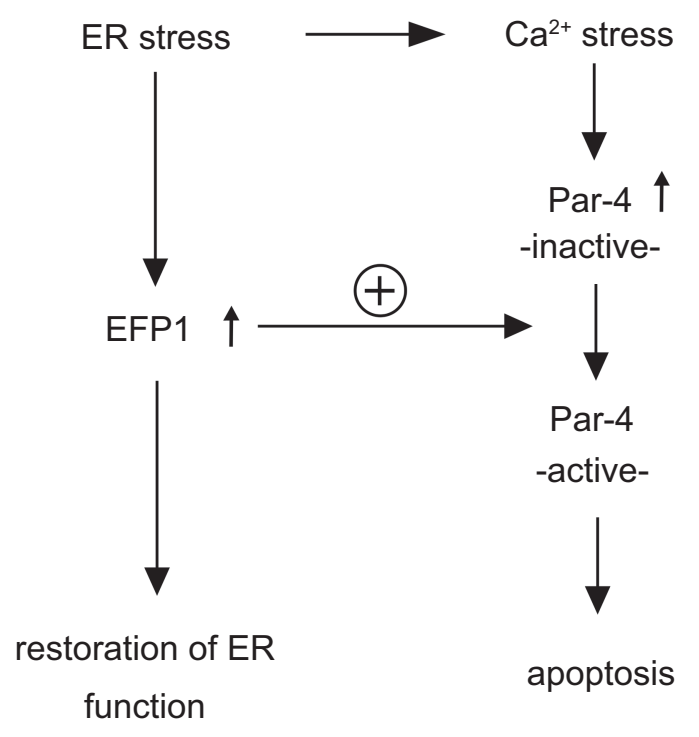

Figure 6 Model for the function of EFPI and Par-4 during ER stress. EFPI is upregulated as part of the ER stress response and contributes to restore the ER function (left part). If the ER stress response fails, an elevated calcium level ("C $\mathrm{a}^{2+}$ stress") might lead to a transcriptional upregulation of Par-4 (right part). Hence, EFPI may function as an activator of Par-4, thus enhancing Par-4-mediated apoptosis.

Abbreviation: ER, endoplasmatic reticulum.

active until ER stress and upregulation of EFP1 occurs. Once fully activated by EFP1, Par-4 induces apoptosis.

By identifying EFP1 as a novel interaction partner and potential regulator of Par-4, we provide a missing link between Par-4 and ER stress, elevated intracellular calcium levels, and the induction of apoptosis. Detailed analysis of the EFP1-Par-4 interplay might therefore yield valuable insights into the regulatory mechanisms of ER stress and apoptosis.

\section{Acknowledgments}

We thank Reinhild Brinker for excellent technical assistance and KH Scheidtmann for helpful discussions. This study was supported by the Deutsche Krebshilfe, Dr Mildred Scheel Stiftung (106365) to UP. The authors report no conflicts of interest in this work.

\section{References}

1. Yoshida H, Haze K, Yanagi H, Yura T, Mori K. Identification of the cis-acting endoplasmic reticulum stress response element responsible for transcriptional induction of mammalian glucose-regulated proteins. Involvement of basic leucine zipper transcription factors. $J$ Biol Chem . 1998;273(50):33741-33749.

2. Haze K, Yoshida H, Yanagi H, Yura T, Mori K. Mammalian transcription factor ATF6 is synthesized as a transmembrane protein and activated by proteolysis in response to endoplasmic reticulum stress. Mol Biol Cell. 1999;10(11):3787-3799.

3. Wang Y, Shen J, Arenzana N, et al. Activation of ATF6 and an ATF6 DNA binding site by the endoplasmic reticulum stress response. $J$ Biol Chem. 2000;275(35):27013-27020.

4. Schröder M, Kaufman RJ. ER stress and the unfolded protein response. Mutat Res. 2005;569(1-2):29-63. 
5. Sitia R, Molteni SN. Stress, protein (mis)folding, and signaling: the redox connection. Sci STKE. 2004;2004(239):pe27.

6. Lucero HA, Lebeche D, Kaminer B. ERcalcistorin/protein-disulfide isomerase acts as a calcium storage protein in the endoplasmic reticulum of a living cell. Comparison with calreticulin and calsequestrin. $J$ Biol Chem. 1998;273(16):9857-9863.

7. Van PN, Peter F, Soling HD. Four intracisternal calcium-binding glycoproteins from rat liver microsomes with high affinity for calcium. No indication for calsequestrin-like proteins in inositol 1,4,5-trisphosphatesensitive calcium sequestering rat liver vesicles. J Biol Chem. 1989; 264(29):17494-17501.

8. Lievremont JP, Rizzuto R, Hendershot L, Meldolesi J. BiP, a major chaperone protein of the endoplasmic reticulum lumen, plays a direct and important role in the storage of the rapidly exchanging pool of $\mathrm{Ca}^{+}$. J Biol Chem. 1997;272(49):30873-30879.

9. Rao RV, Ellerby HM, Bredesen DE. Coupling endoplasmic reticulum stress to the cell death program. Cell Death Differ. 2004;11(4):372-380.

10. Cryns V, Yuan J. Proteases to die for. Genes Dev. 1998;12(11):1551-1570

11. Hitomi J, Katayama T, Eguchi Y, et al. Involvement of caspase-4 in endoplasmic reticulum stress-induced apoptosis and Abeta-induced cell death. J Cell Biol. 2004;165(3):347-356.

12. Nakagawa $T$, Zhu H, Morishima N, et al. Caspase-12 mediates endoplasmic-reticulum-specific apoptosis and cytotoxicity by amyloid-beta Nature. 2000;403(6765):98-103.

13. Jimbo A, Fujita E, Kouroku Y, et al. ER stress induces caspase-8 activation, stimulating cytochrome $\mathrm{c}$ release and caspase-9 activation. Exp Cell Res. 2003;283(2):156-166.

14. Rao RV, Hermel E, Castro-Obregon S, et al. Coupling endoplasmic reticulum stress to the cell death program. Mechanism of caspase activation. J Biol Chem. 2001;276(36):33869-33874.

15. Obeng EA, Boise LH. Caspase- 12 and caspase- 4 are not required for caspase-dependent endoplasmic reticulum stress-induced apoptosis. J Biol Chem. 2005;280(33):29578-29587.

16. Wang XZ, Lawson B, Brewer JW, et al. Signals from the stressed endoplasmic reticulum induce C/EBP-homologous protein (CHOP/ GADD153). Mol Cell Biol. 1996;16(8):4273-4280.

17. McCullough KD, Martindale JL, Klotz LO, Aw TY, Holbrook NJ. Gadd153 sensitizes cells to endoplasmic reticulum stress by downregulating Bcl2 and perturbing the cellular redox state. Mol Cell Biol. 2001;21(4):1249-1259.

18. Mohamad N, Gutierrez A, Nunez M, et al. Mitochondrial apoptotic pathways. Biocell. 2005;29(2):149-161.

19. Mattson MP, Gary DS, Chan SL, Duan W. Perturbed endoplasmic reticulum function, synaptic apoptosis and the pathogenesis of Alzheimer's disease. Biochem Soc Symp. 2001; (67):151-162.

20. Sells SF, Wood DP, Jr, Joshi-Barve SS, et al. Commonality of the gene programs induced by effectors of apoptosis in androgen-dependent and independent prostate cells. Cell Growth Differ. 1994;5(4):457-466.

21. Boosen M, Vetterkind S, Koplin A, Illenberger S, Preuss U. Par-4mediated recruitment of Amida to the actin cytoskeleton leads to the induction of apoptosis. Exp Cell Res. 2005;311(2):177-191.

22. Page G, Kögel D, Rangnekar V, Scheidtmann KH. Interaction partners of Dlk/ZIP kinase: co-expression of Dlk/ZIP kinase and Par-4 results in cytoplasmic retention and apoptosis. Oncogene. 1999;18(51) 7265-7273.

23. Vetterkind S, Illenberger S, Kubicek J, et al. Binding of Par-4 to the actin cytoskeleton is essential for Par-4/Dlk-mediated apoptosis. Exp Cell Res. 2005;305(2):392-408.

24. Cheema SK, Mishra SK, Rangnekar VM, et al. Par-4 transcriptionally regulates Bcl-2 through a WT1-binding site on the bcl-2 promoter. J Biol Chem. 2003;278(22):19995-20005.

25. Goswami A, Burikhanov R, de Thonel A et al. Binding and phosphorylation of par-4 by akt is essential for cancer cell survival. Mol Cell. 2005;20(1):33-44

26. Wang D, De Deken X, Milenkovic M, et al. Identification of a novel partner of duox: EFP1, a thioredoxin-related protein. J Biol Chem. 2005;280(4):3096-3103.
27. Klawitz I, Preuss U, Scheidtmann KH. Interaction of SV40 large $\mathrm{T}$ antigen with components of the nucleo/cytoskeleton. Int $J$ Oncol. 2001;19(6):1325-1332.

28. Short JM, Fernandez JM, Sorge JA, Huse WD. Lambda ZAP: a bacteriophage lambda expression vector with in vivo excision properties. Nucleic Acids Res. 1988;16(15):7583-7600.

29. Sells SF, Han SS, Muthukkumar S, et al. Expression and function of the leucine zipper protein Par-4 in apoptosis. Mol Cell Biol. 1997; 17(7):3823-3832.

30. Chenna R, Sugawara H, Koike T, et al. Multiple sequence alignment with the Clustal series of programs. Nucleic Acids Res. 2003;31(13): 3497-3500.

31. Rost B, Yachdav G, Liu J. The PredictProtein server. Nucleic Acids Res. 2004;32(Web Server issue):W321-326.

32. Berger B, Wilson DB, Wolf E, et al. Predicting coiled coils by use of pairwise residue correlations. Proc Natl Acad Sci U S A. 1995; 92(18):8259-8263.

33. Horton P, Nakai K. Better prediction of protein cellular localization sites with the k nearest neighbors classifier. Proc Int Conf Intell Syst Mol Biol. 1997;5:147-152.

34. Blom N, Gammeltoft S, Brunak S. Sequence and structure-based prediction of eukaryotic protein phosphorylation sites. J Mol Biol. 1999;294(5):1351-1362.

35. Bendtsen JD, Nielsen H, von Heijne G, Brunak S. Improved prediction of signal peptides: SignalP 3.0. J Mol Biol. 2004;340(4):783-795.

36. Kawai T, Akira S, Reed JC. ZIP kinase triggers apoptosis from nuclear PML oncogenic domains. Mol Cell Biol. 2003;23(17):6174-6186.

37. Holmgren A. Thioredoxin catalyzes the reduction of insulin disulfides by dithiothreitol and dihydrolipoamide. J Biol Chem. 1979;254(19): 9627-9632.

38. Kappler R, Pietsch T, Weggen S, Wiestler OD, Scherthan H. Chromosomal imbalances and DNA amplifications in SV40 large $\mathrm{T}$ antigen-induced primitive neuroectodermal tumor cell lines of the rat. Carcinogenesis. 1999;20(8):1433-1438.

39. Martin JL. Thioredoxin - a fold for all reasons. Structure. 1995;3(3): $245-250$.

40. Holmgren A, Soderberg BO, Eklund H, Branden CI. Three-dimensional structure of Escherichia coli thioredoxin-S2 to 2.8 A resolution. Proc Natl Acad Sci U S A. 1975;72(6):2305-2309.

41. Ellgaard L, Ruddock LW. The human protein disulphide isomerase family: substrate interactions and functional properties. EMBO Rep. 2005;6(1):28-32.

42. Ferrari DM, Soling HD. The protein disulphide-isomerase family: unravelling a string of folds. Biochem J. 1999;339 ( Pt 1):1-10.

43. Darby NJ, Penka E, Vincentelli R. The multi-domain structure of protein disulfide isomerase is essential for high catalytic efficiency. J Mol Biol. 1998;276(1):239-247.

44. Barnewitz K, Guo C, Sevvana M, et al. Mapping of a substrate binding site in the protein disulfide isomerase-related chaperone wind based on protein function and crystal structure. J Biol Chem. 2004;279(38): 39829-39837.

45. Horibe T, Gomi M, Iguchi D, et al. Different contributions of the three CXXC motifs of human protein-disulfide isomerase-related protein to isomerase activity and oxidative refolding. $J$ Biol Chem. 2004;279(6):4604-4611.

46. Boyce M, Yuan J. Cellular response to endoplasmic reticulum stress: a matter of life or death. Cell Death Differ. 2006;13(3):363-373.

47. Turano C, Coppari S, Altieri F, Ferraro A. Proteins of the PDI family: unpredicted non-ER locations and functions. $J$ Cell Physiol. 2002;193(2):154-163.

48. Gerner C, Holzmann K, Meissner M, et al. Reassembling proteins and chaperones in human nuclear matrix protein fractions. $J$ Cell Biochem. 1999;74(2):145-151.

49. Terada K, Manchikalapudi $P$, Noiva R, et al. Secretion, surface localization, turnover, and steady state expression of protein disulfide isomerase in rat hepatocytes. J Biol Chem. 1995;270(35): 20410-20416 
50. Hirano N, Shibasaki F, Sakai R, et al. Molecular cloning of the human glucose-regulated protein ERp57/GRP58, a thiol-dependent reductase. Identification of its secretory form and inducible expression by the oncogenic transformation. Eur J Biochem. 1995;234(1):336-342.

51. Lewis MJ, Mazzarella RA, Green M. Structure and assembly of the endoplasmic reticulum: biosynthesis and intracellular sorting of ERp61, ERp59, and ERp49, three protein components of murine endoplasmic reticulum. Arch Biochem Biophys. 1986;245(2):389-403.

52. Hsu SC, Kirschenbaum F, Miller J, Cordell B, McCarthy JV. Structural and functional characterization of the upstream regulatory region of the human gene encoding prostate apoptosis response factor-4. Gene. 2002;295(1):109-116.

53. Gurumurthy S, Goswami A, Vasudevan KM, Rangnekar VM. Phosphorylation of Par-4 by protein kinase A is critical for apoptosis. Mol Cell Biol. 2005;25(3):1146-1161.

54. Xie J, Chang X, Zhang X, Guo Q. Aberrant induction of Par-4 is involved in apoptosis of hippocampal neurons in presenilin-1 M146V mutant knock-in mice. Brain Res. 2001;915(1):1-10.

55. Xie J, Guo Q. PAR-4 is involved in regulation of beta-secretase cleavage of the Alzheimer amyloid precursor protein. J Biol Chem. 2005;280(14):13824-13832.
56. Guo Q, Fu W, Xie J, et al. Par-4 is a mediator of neuronal degeneration associated with the pathogenesis of Alzheimer disease. Nat Med. 1998; 4(8):957-962.

57. Guo Q, Xie J. AATF inhibits aberrant production of amyloid beta peptide 1-42 by interacting directly with Par-4. J Biol Chem. 2004; 279(6):4596-4603.

58. Xie J, Awad KS, Guo Q. RNAi knockdown of Par-4 inhibits neurosynaptic degeneration in ALS-linked mice. J Neurochem. 2005;92(1): 59-71.

59. Pedersen WA, Luo H, Kruman I, Kasarskis E, Mattson MP. The prostate apoptosis response-4 protein participates in motor neuron degeneration in amyotrophic lateral sclerosis. FASEB J. 2000;14(7): 913-924.

60. Culmsee C, Zhu Y, Krieglstein J, Mattson MP. Evidence for the involvement of Par-4 in ischemic neuron cell death. J Cereb Blood Flow Metab. 2001;21(4):334-343.

61. Duan W, Zhang Z, Gash DM, Mattson MP. Participation of prostate apoptosis response-4 in degeneration of dopaminergic neurons in models of Parkinson's disease. Ann Neurol. 1999;46(4): 587-597.
Cell Health and Cytoskeleton

\section{Publish your work in this journal}

Cell Health and Cytoskeleton is an international, peer-reviewed open access journal focusing on all aspects of cell structure and function contributing to normal physiology and cell health and exploring the pathogenesis of cell dysfunction leading to adverse conditions and disease in the organism. The journal welcomes papers covering original research,

\section{Dovepress}

basic science, reviews and evaluations, guidelines, expert opinion and commentary, case reports and extended reports. The manuscript management system is completely online and includes a very quick and fair peerreview system, which is all easy to use. Visit http://www.dovepress.com/ testimonials.php to read real quotes from published authors. 